\title{
Bronchoscopic Microsampling for Bacterial Colony Counting in Relevant Lesions in Patients with Pulmonary Mycobacterium avium Complex Infection
}

\author{
Atsuhito Ushiki ${ }^{1}$, Yoshitaka Yamazaki ${ }^{2}$, Sekiya Koyama ${ }^{3}$, Kenji Tsushima $^{1}$, \\ Hiroshi Yamamoto ${ }^{1}$, Masayuki Hanaoka ${ }^{1}$ and Keishi Kubo ${ }^{1}$
}

\begin{abstract}
Objective The incidence of pulmonary Mycobacterium avium complex (MAC) infections with nodular/ bronchiectasis lesions is increasing. However, factors determining deterioration are unknown. In the present study, we investigated quantitative MAC cultures obtained through bronchoscopic microsampling (BMS) from patients with pulmonary MAC infection and analyzed the relationship between MAC culture and the short-term natural history. We also assessed chest computed tomography (CT) findings for the deteriorating factors.

Design For this prospective study, MAC was collected from peripheral lung lesions by BMS through endobronchial ultrasonography. MAC colonies were counted on Middlebrook 7H11 agar. We compared the number of MAC colonies with laboratory data and chest CT findings.

Patients We studied 26 patients with pulmonary MAC infection.

Results The patients were divided into 2 groups: 11 patients in the non-deteriorated group and 15 patients in the deteriorated group. The number of MAC colonies was significantly correlated with deterioration of MAC infection $(\mathrm{p}<0.001)$. In the non-deteriorated group, chest CT scans showed nodular/bronchiectasis lesions in 8 patients $(73 \%)$ and consolidated lesions in 3 patients $(27 \%)$. In the deteriorated group, chest CT scans showed nodular/bronchiectasis lesions in 1 patient $(7 \%)$, consolidated lesions in 6 patients $(40 \%)$, and cavitary lesions in 8 patients $(53 \%)$.

Conclusion The number of MAC colonies in relevant lesions investigated by BMS was significantly larger in the deteriorated group than in the non-deteriorated group. Cavitary and consolidated lesions observed from chest CT scans are thought to indicate a high risk of progression of pulmonary MAC infection.
\end{abstract}

Key words: non-tuberculous mycobacteriosis, Mycobacterium avium complex, bronchoscopic microsampling, endobronchial ultrasonography

(Intern Med 50: 1287-1292, 2011)

(DOI: 10.2169/internalmedicine.50.5034)

\section{Introduction}

Pulmonary Mycobacterium avium complex (MAC), which includes $M$. avium and $M$. intracellulare infection, occurs in patients without predisposing lung diseases. The number of cases has risen in recent years (1-3). Chest computed tomography (CT) scans of MAC infection show the character- istic findings, such as multiple small nodules, bronchiectasis, consolidation and cavitary lesions distributed mainly in the upper, middle, and lingular lobes (2-4). Early studies have shown that pulmonary MAC infections in symptomatic (and some apparently asymptomatic) patients can be fatal (5). On the other hand, Thomson and Yew documented cases where disease remitted spontaneously (6). Wallace et al. found that semiquantitative (0-4+) cultures of MAC colony counts on

${ }^{1}$ The First Department of Internal Medicine, Shinshu University School of Medicine, Japan, ${ }^{2}$ Department of Infectious Disease, Suzaka Hospital, Japan and ${ }^{3}$ Department of Respiratory Medicine, Chushin Matsumoto Hospital, Japan Received for publication December 20, 2010; Accepted for publication March 1, 2011 Correspondence to Dr. Yoshitaka Yamazaki, yamazakiy@ suzakahp.jp 
solid media are useful for assessing responses to therapy (7). However, it is unclear if this approach is useful for predicting the short-term natural history of pulmonary MAC infection.

A bronchoscopic microsampling (BMS) probe was developed to sample biochemical constituents of the local bronchial epithelial lining fluid (ELF) (8). BMS is performed while observing the bronchial lumen using a bronchofiberscope. When the target site is reached, the inner probe is advanced slowly into the distal airway to absorb bronchial ELF. BMS is useful for detecting the biochemical constituents in acute respiratory distress syndrome $(8,9)$ and respiratory tract infection $(10,11)$ and for detecting antibiotic concentrations $(12,13)$, tumor markers of small peripheral lung carcinoma (14), and gene expression in pulmonary nodules (15). In addition, Sasabayashi et al. reported that BMS can be used for quantitative sampling of bacteria in vitro (16). Recently developed small-caliber ultrasound probes have enabled a wider clinical application of ultrasonography (US) for evaluating tracheal and bronchial lesions. Endobronchial US (EBUS) has been used for imaging guidance in transbronchial approaches to peripheral pulmonary lesions (17). Thus, utilizing EBUS with a guide sheath (EBUS-GS) increases the ability to endoscopically diagnose peripheral pulmonary lesions (18).

In the present study, we used BMS guided EBUS-GS to assess the bacteria number in relevant lesions in patients with MAC infection and analyzed the correlation of the number of MAC colonies with deterioration and chest CT findings.

\section{Study Population and Methods}

\section{1) Patients}

We studied 26 patients with pulmonary MAC infection, the diagnosis of which was based on criteria of the American Thoracic Society/Infectious Disease of America (19). All patients visited our hospital because of cough and/or sputum and abnormal findings on chest radiographs and CT scans, such as multiple centrilobular small nodules, bronchiectasis, consolidation, and/or cavities suggesting MAC infection. All patients had nodular/bronchiectasis disease (19). None of the patients had previously received treatment for pulmonary MAC infection. Written informed consent was obtained from all patients before inclusion in the study, which was approved by the Ethics Committee of the Shinshu University School of Medicine.

\section{2) Laboratory data and chest CT scan}

Clinical data, including age, body mass index (BMI), and laboratory tests, including those for white blood cell count (WBC), lymphocyte count (LYM), total protein (TP), albu$\min (\mathrm{ALB}), \mathrm{C}$-reactive protein (CRP), and the erythrocyte sedimentation rate (ESR), were collected for each patient. We also used high-resolution CT scans to asses all patients.
All tables represent data collected at baseline, when pulmonary MAC infection was suspected.

\section{3) EBUS-GS and BMS procedures}

After routine premedications, a flexible 1T-260 fiberoptic bronchoscope (Olympus, Tokyo, Japan) was inserted beyond the vocal cords so that all segments of the bronchial tree were visualized. Based on the radiographic findings, a curette that had been introduced into the guide sheath was maneuvered into the bronchus of interest. After the angulated curette was advanced into the bronchus leading to the lesions, a fluorescent radiograph was taken to confirm that the curette had reached the lesions. The curette was then withdrawn, leaving the guide sheath in place.

An ultrasound probe $(20 \mathrm{MHz}$, mechanical-radial type, UM-S30-20R; Olympus) connected to an endoscopic ultrasound system (EU-M2000; Olympus) was introduced into the guide sheath until the tip of the probe extended to the far end of the guide sheath to confirm that the BMS probe had been inserted into the relevant lesion to collect ELF from the same site.

A polyethylene probe (length, $30 \mathrm{~mm}$; diameter, $1.2 \mathrm{~mm}$ ) attached to the tip of the BMS device (BC-401C; Olympus) was introduced into the guide sheath, used to absorb samples from relevant lesions, and stored back in the sheath after 10 seconds. The BMS device was then removed from the guide sheath, and the probe was cut with disinfected scissors and placed in $1 \mathrm{~mL}$ normal saline in an Eppendorf tube. This sampling procedure was repeated thrice.

Following EBUS-GS and BMS sampling, bronchial washing was performed by instilling $20 \mathrm{~mL}$ of $0.9 \%$ normal saline at room temperature through the internal channel of the bronchoscope and aspirated into a trap connected to suction tubing. On average, 3-5 mL fluid was retrieved in the suction trap.

The patients were monitored using continuous pulse oximetry and electrocardiography during the bronchoscopy.

\section{4) MAC culture}

The Eppendorf tube was stirred for 1 minute using a vortex. Serial dilutions (1:10) were prepared and inoculated $(100 \mu \mathrm{L})$ on Middlebrook 7H11 agar (Becton Dickinson, Sparks, MD, USA). MAC was cultured at $37^{\circ} \mathrm{C}$ in $5 \% \mathrm{CO}_{2}$ for 4 weeks before the colonies were counted.

\section{5) Estimation of MAC deterioration}

Deterioration of infection was estimated mainly on the basis of chest CT scans. Chest CT was performed at intervals of 4 months after BMS without treatment. The CT scans were evaluated by 2 chest radiologists who were unaware of the patients' clinical course. After a mean observation period of $11 \pm 3$ months (4 to 12 months), 26 patients were divided into 2 groups: the deteriorated group and the non-deteriorated group. Deterioration was defined as follows: appearance of new lesions, increase in the number of the nodules, progression of bronchiectasis, or expansion of 
Table 1. Clinical Features of Patients with Pulmonary MAC Infection

\begin{tabular}{lccc} 
& & \multicolumn{2}{c}{ Data are mean $\pm \mathrm{SD}$} \\
\hline & $\begin{array}{c}\text { Non-deteriorated } \\
\text { group } \\
(\mathrm{n}=11)\end{array}$ & $\begin{array}{c}\text { Deteriorated } \\
\text { group } \\
(\mathrm{n}=15)\end{array}$ & $\mathrm{p}$ \\
\hline Age $(\mathrm{y})$ & $67 \pm 10$ & $67 \pm 9$ & $\mathrm{NS}$ \\
Sex $(\mathrm{female} / \mathrm{male})$ & $9 / 2$ & $14 / 1$ & $\mathrm{NS}$ \\
BMI $\left(\mathrm{kg} / \mathrm{m}^{2}\right)$ & $20.0 \pm 2.2$ & $18.7 \pm 1.7$ & $\mathrm{NS}$ \\
WBC $(\mathrm{cells} / \mu \mathrm{L})$ & $5464 \pm 1608$ & $6938 \pm 2882$ & $\mathrm{NS}$ \\
Lymphocyte $(\mathrm{cells} / \mu \mathrm{L})$ & $1517 \pm 410$ & $1472 \pm 643$ & $\mathrm{NS}$ \\
Total protein $(\mathrm{g} / \mathrm{dL})$ & $7.3 \pm 0.7$ & $7.5 \pm 0.4$ & $\mathrm{NS}$ \\
Albumin $(\mathrm{g} / \mathrm{dL})$ & $4.3 \pm 0.2$ & $4.0 \pm 0.4$ & $\mathrm{NS}$ \\
CRP $(\mathrm{mg} / \mathrm{dL})$ & $0.20 \pm 0.26$ & $1.23 \pm 2.00$ & $\mathrm{NS}$ \\
ESR $(\mathrm{mm} / \mathrm{h})$ & $19 \pm 14$ & $38 \pm 29$ & $\mathrm{NS}$ \\
\hline MAC: Mycobacterium avium complex; BMI: body mass index; WBC: white blood cell; \\
CRP: C-reactive protein; ESR: erythrocyte sedimentation rate; NS: not significant
\end{tabular}

Table 2. Clinical Findings and the Number of MAC Colonies in the Deteriorated Group of Patients with Pulmonary MAC Infection

\begin{tabular}{cccccccc}
\hline $\begin{array}{c}\text { Patient } \\
\text { No. }\end{array}$ & $\begin{array}{c}\text { Age } \\
\text { (y) }\end{array}$ & Sex & Symptoms & Chest CT findings & $\begin{array}{c}\text { EBUS } \\
\text { findings }\end{array}$ & $\begin{array}{c}\text { BMS MAC } \\
(\mathrm{CFU} / \mathrm{mL})\end{array}$ & $\begin{array}{c}\text { Washing MAC } \\
(\mathrm{CFU} / \mathrm{mL})\end{array}$ \\
\hline 1 & 76 & $\mathrm{M}$ & sputum & cavitary & low and iso & $1.7 \times 10^{4}$ & ND \\
2 & 55 & $\mathrm{~F}$ & sputum & cavitary & low and iso & $1.3 \times 10^{5}$ & $\begin{array}{c}\text { ND } \\
\text { consolidation }\end{array}$ \\
3 & 68 & $\mathrm{~F}$ & bloody sputum & iso & $1.1 \times 10^{5}$ & $2.0 \times 10^{5}$ \\
4 & 56 & $\mathrm{~F}$ & cough & consolidation & iso & $1.0 \times 10^{4}$ & $5.8 \times 10^{4}$ \\
5 & 59 & $\mathrm{~F}$ & sputum & consolidation & iso & $6.0 \times 10^{3}$ & $1.8 \times 10^{4}$ \\
6 & 71 & $\mathrm{~F}$ & cough & consolidation & iso & $3.0 \times 10^{4}$ & $5.3 \times 10^{4}$ \\
7 & 76 & $\mathrm{~F}$ & cough & cavitary & low and iso & $3.1 \times 10^{6}$ & $2.4 \times 10^{3}$ \\
8 & 66 & $\mathrm{~F}$ & cough & cavitary & low and iso & $1.7 \times 10^{5}$ & $2.7 \times 10^{5}$ \\
9 & 59 & $\mathrm{~F}$ & bloody sputum & cavitary & low and iso & $8.6 \times 10^{4}$ & $7.0 \times 10^{3}$ \\
10 & 71 & $\mathrm{~F}$ & cough & cavitary & low and iso & $3.7 \times 10^{5}$ & $8.4 \times 10^{4}$ \\
11 & 83 & $\mathrm{~F}$ & bloody sputum & cavitary & low and iso & $5.0 \times 10^{4}$ & $1.5 \times 10^{5}$ \\
12 & 79 & $\mathrm{~F}$ & cough & cavitary & low and iso & $2.9 \times 10^{6}$ & $3.3 \times 10^{4}$ \\
13 & 58 & $\mathrm{~F}$ & sputum & consolidation & iso & $3.4 \times 10^{4}$ & $4.0 \times 10^{3}$ \\
14 & 73 & $\mathrm{~F}$ & sputum & consolidation & iso & $2.5 \times 10^{5}$ & $6.9 \times 10^{6}$ \\
15 & 59 & $\mathrm{~F}$ & bloody sputum & nodular/bronchiectasis & heterogeneous & $7.1 \times 10^{3}$ & $1.8 \times 10^{4}$ \\
\hline
\end{tabular}

CT: computed tomography; BMS: bronchial microsampling; MAC: Mycobacterium avium complex; CFU: colony forming unit; EBUS: endobronchial ultrasonography; ND: not done

consolidation or cavity. If a chest CT scan showed improvement for 1 lesion and progression for the other lesions, we defined the case as not deteriorated.

\section{6) Statistical analysis}

All results, except for the number of MAC colonies, were expressed as mean $\pm \mathrm{SD}$. The number of MAC colonies was expressed as median figures and ranges. Clinical features, laboratory data, and the number of MAC colonies between the 2 groups were compared using an unpaired $t$-test. The difference in sex and symptoms between the 2 groups was compared using Fisher's exact test. Dunnett's test was used for comparison of the number of MAC colonies among three groups. Correlation between deterioration and the number of MAC colonies was assessed by Fisher's exact test. Correlations between the number of MAC colonies collected by BMS and those collected by bronchial washing and clinical features were examined using Pearson's product-moment correlation coefficient. A $p$ value of $<0.05$ indicated a significant difference.

\section{Results}

\section{1) Patient characteristics and laboratory data}

We studied 26 patients who had no underlying disease. The patients were divided into 2 groups based on chest CT findings: 11 patients (9 females) in the non-deteriorated group and 15 patients (14 females) in the deteriorated group. Laboratory data for all patients did not change at the end of deterioration. Laboratory data were not significantly different between the 2 groups (Table 1). There were significantly more patients with bloody sputum in the deteriorated group (Table 2).

\section{2) Number of MAC colonies}

The number of MAC colonies collected by BMS is shown in Table 2, 3. There was no correlation between the number of MAC colonies collected by BMS and clinical features (age, BMI, WBC, LYM, TP, ALB, CRP, and ESR). The number of MAC colonies collected by BMS was significantly larger in the deteriorated group $\left(6.0 \times 10^{3}-3.1 \times 10^{6}\right.$; me- 
Table 3. Clinical Findings and the Number of MAC Colonies in The-non Deteriorated Group of Patients with Pulmonary MAC Infection

\begin{tabular}{|c|c|c|c|c|c|c|c|}
\hline $\begin{array}{l}\text { Patient } \\
\text { No. }\end{array}$ & $\begin{array}{l}\text { Age } \\
\text { (y) }\end{array}$ & Sex & Symptoms & Chest CT findings & $\begin{array}{l}\text { EBUS } \\
\text { findings }\end{array}$ & $\begin{array}{c}\text { BMS MAC } \\
(\mathrm{CFU} / \mathrm{mL})\end{array}$ & $\begin{array}{c}\text { Washing MAC } \\
(\mathrm{CFU} / \mathrm{mL})\end{array}$ \\
\hline 1 & 83 & M & cough & consolidation & iso & $5.1 \times 10^{3}$ & ND \\
\hline 2 & 51 & $\mathrm{~F}$ & cough & nodular/bronchiectasis & heterogeneous & $5.5 \times 10^{3}$ & $2.8 \times 10^{3}$ \\
\hline 3 & 76 & M & sputum & nodular/bronchiectasis & heterogeneous & $5.0 \times 10^{2}$ & $1.0 \times 10^{3}$ \\
\hline 4 & 72 & $\mathrm{~F}$ & cough & nodular/bronchiectasis & heterogeneous & $1.0 \times 10^{2}$ & $1.1 \times 10^{4}$ \\
\hline 5 & 75 & $\mathrm{~F}$ & sputum & consolidation & iso & $2.3 \times 10^{3}$ & $3.4 \times 10^{2}$ \\
\hline 6 & 59 & $\mathrm{~F}$ & cough & nodular/bronchiectasis & heterogeneous & $3.0 \times 10$ & $1.0 \times 10$ \\
\hline 7 & 56 & $\mathrm{~F}$ & cough & nodular/bronchiectasis & heterogeneous & $5.0 \times 10^{2}$ & $3.0 \times 10^{2}$ \\
\hline 8 & 72 & $\mathrm{~F}$ & cough & nodular/bronchiectasis & heterogeneous & $1.5 \times 10^{3}$ & $3.9 \times 10^{3}$ \\
\hline 9 & 63 & $\mathrm{~F}$ & sputum & consolidation & iso & $1.3 \times 10^{3}$ & $2.8 \times 10^{3}$ \\
\hline 10 & 61 & $\mathrm{~F}$ & sputum & nodular/bronchiectasis & heterogeneous & $4.7 \times 10^{3}$ & $1.0 \times 10$ \\
\hline 11 & 66 & $\mathrm{~F}$ & sputum & nodular/bronchiectasis & heterogeneous & $1.0 \times 10^{2}$ & $2.3 \times 10^{3}$ \\
\hline
\end{tabular}

CT: computed tomography; BMS: bronchial microsampling; MAC: Mycobacterium avium complex; CFU: colony forming unit; EBUS endobronchial ultrasonography; ND: not done

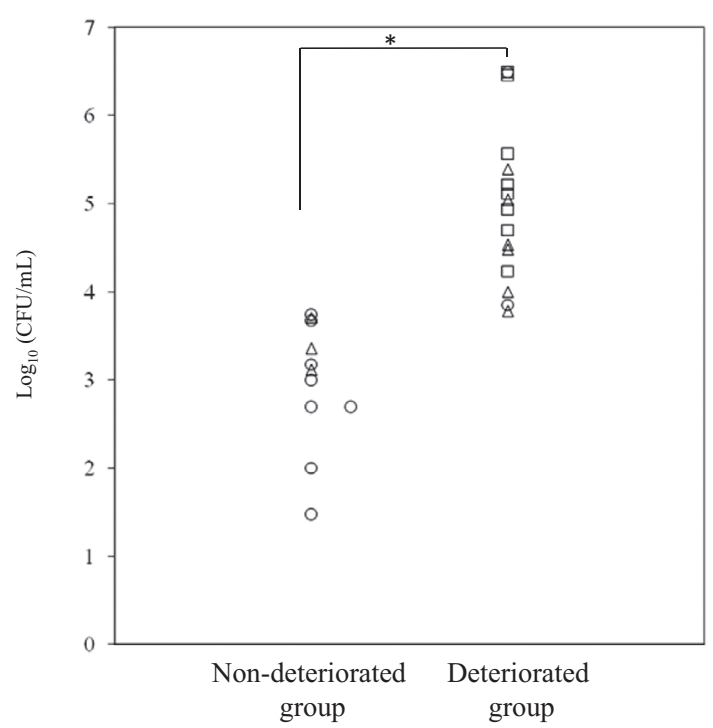

Figure 1. The number of MAC colonies in ELF collected by BMS under EBUS-GS. The number of MAC colonies in the deteriorated group $(n=15)$ was significantly larger than that in the non-deteriorated group $(\mathrm{n}=11)$. ${ }^{*} \mathbf{p}<\mathbf{0 . 0 0 1}$. Circle, nodular/ bronchiectasis lesion; triangle, consolidated lesion; square, cavitary lesion. Fisher's exact test showed a significant correlation between the number of MAC colonies and the deteriorated group $(\mathbf{p}<0.001)$.

dian: $8.6 \times 10^{4}$ colony forming units $\left.(\mathrm{CFU}) / \mathrm{mL}\right)$ than in the non-deteriorated group $\left(3.0 \times 10-5.5 \times 10^{3}\right.$; median: $1.3 \times 10^{3}$ $\mathrm{CFU} / \mathrm{mL})$, as shown in Fig. 1 ( $\mathrm{p}<0.001)$.

The number of MAC colonies collected by BMS was significantly larger in cavitary lesions $\left(1.7 \times 10^{4}-3.1 \times 10^{6}\right.$; median: $1.5 \times 10^{5} \mathrm{CFU} / \mathrm{mL}$ ) than in nodular/bronchiectasis lesions $\left(3.0 \times 10-7.1 \times 10^{3}\right.$; median: $\left.1.0 \times 10^{3} \mathrm{CFU} / \mathrm{mL} ; \mathrm{p}<0.05\right)$. The difference between the number of MAC colonies collected by BMS in consolidated lesions $\left(1.3 \times 10^{3}-2.5 \times 10^{5}\right.$; median: $1.0 \times 10^{4} \mathrm{CFU} / \mathrm{mL}$ ) and those in nodular/bronchiectasis lesions and cavitary lesions was not significant (Fig. 2).

The number of MAC colonies collected by bronchial washing is shown in Table 2, 3. Although we counted no colonies in 3 cases, the number of MAC colonies collected

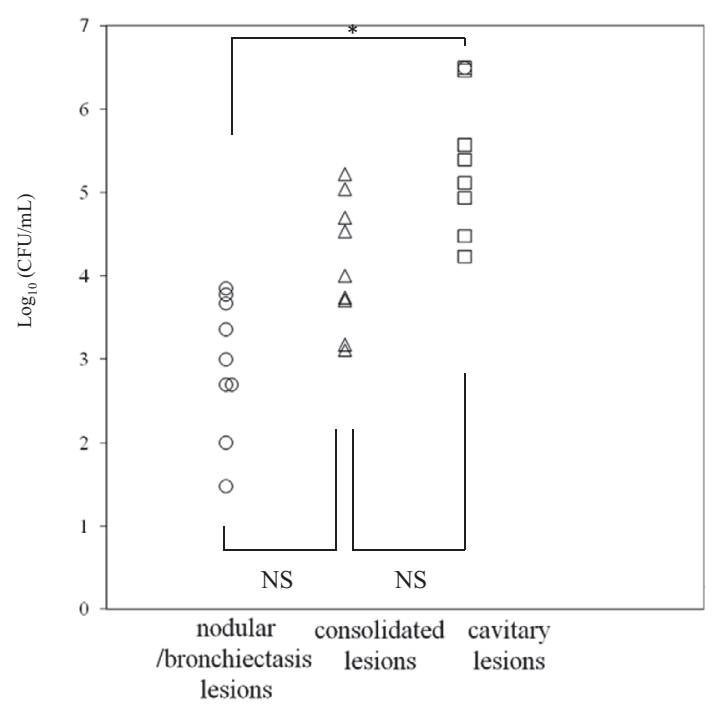

Figure 2. The number of MAC colonies collected by BMS under EBUS-GS. The number of MAC colonies in the cavitary lesions $(n=8)$ was larger than that in the nodular/bronchiectasis lesions $(n=9)$. * $\mathbf{p}<0.05$. The number of MAC colonies between nodular/bronchiectasis lesions and consolidated lesions $(n=9)$ was not significantly different. The number of MAC colonies between consolidated lesions and cavitary lesions was also not significantly different. NS: not significant

by bronchial washing was significantly larger in the deteriorated group $\left(2.4 \times 10^{3}-6.9 \times 10^{6}\right.$; median: $\left.5.3 \times 10^{4} \mathrm{CFU} / \mathrm{mL}\right)$ than in the non-deteriorated group $\left(1.0 \times 10-1.1 \times 10^{4}\right.$; median: $\left.1.7 \times 10^{3} \mathrm{CFU} / \mathrm{mL} ; \mathrm{p}<0.001\right)$. The patient recording $>1.0 \times 10^{4}$ $\mathrm{CFU} / \mathrm{mL}$ MAC colonies was significantly correlated with the deteriorated group $(\mathrm{p}<0.05)$ rather than the nondeteriorated group. However, the difference in the number of MAC colonies collected by bronchial washing among nodular/bronchiectasis lesions, consolidated lesions and cavitary lesions was not significant. Furthermore, the number of MAC colonies collected by bronchial washing did not correlate with that collected by BMS $(r=-0.02)$. 


\section{3) Chest CT scan findings and EBUS}

The chest CT scans displayed 3 patterns. The first pattern consisted of multiple centrilobular small nodules and bronchiectasis distributed mainly in the upper, middle, and lingular lobes (nodular/bronchiectasis lesions), while EBUS demonstrated heterogeneous internal echoes and unclear margins around the lesions. The second pattern exhibited consolidation and/or collapse (consolidated lesions) with nodular/ bronchiectasis lesions, and EBUS recorded iso-echoes and clear margins around the lesions. The third pattern consisted of single or multiple thin-wall cavities (cavitary lesions) with nodular/bronchiectasis, while EBUS demonstrated lowechoes surrounded by iso-echoes.

Chest CT scans in the non-deteriorated group (Table 3) showed nodular/bronchiectasis lesions in 8 patients $(73 \%)$ and consolidated lesions in 3 patients $(27 \%)$; cavitary patterns were not included. In the deteriorated group (Table 2), chest CT scans showed nodular/bronchiectasis lesions in 1 patient $(7 \%)$, consolidated lesions in 6 patients $(40 \%)$ and cavitary lesions in 8 patients $(53 \%)$.

\section{Discussion}

In the present study, we counted the number of MAC colonies collected from relevant lesions in patients with pulmonary MAC infections using a BMS probe. EBUS imaging of peripheral lung cancer results in a definite hypoechoic or iso-echoic signal that reflects solid tissue $(17,20)$. Our study showed EBUS that imaging reflected heterogeneous patterns of the relevant lesions. Solid lesions, including small nodules, collapse, infiltration and cavity walls, showed hypoechoic or iso-echoic signals as has been reported. Thus, we concluded that samples from relevant lesions were accurately collected using EBUS.

Bronchoalveolar lavage (BAL) and protected specimen brushes (PSB) were used for collecting pathogenic organisms (21-23). However, BAL may carry the risk of subsequent complications, e.g., transient hypoxia or intrapulmonary dissemination of pathogenic organisms $(24,25)$. A 10fold variation in specimen size collected by PSB would yield a difference of only $1 \log$ unit with quantitative bacterial counts (16). Sasabayashi et al. reported that their BMS probe was capable of repeatedly collecting MAC without altering bacterial concentration (16). This may explain the lack of correlation between MAC colony numbers from BAL and BMS collections.

Radiological findings of nodular/bronchiectasis disease showed centrilobular nodules in the outer zone of the middle lobe and lingula fields, associated with dilatation and thickening of the draining small bronchi. Cavitation and consolidation frequently accompany these findings $(19,26,27)$. The pathological examination of the only nodular/bronchiectasis lesions showed peribronchial infiltration of mononuclear cells and epithelioid cells surrounding a bronchus or a bronchiole, but the alveolar region was almost nor- mal $(27,28)$. On the other hand, the pathological examination of consolidation lesions showed inflammatory thickening of the alveolar walls, loosely grounded granulomas, and/ or coalescent inflammatory infiltrates completely replacing the normal alveoli (27) and that of cavitary lesions showed caseous materials, epithelioid cells with multinucleated giant cells, granulation tissue, and a fibrous capsule had replaced the normal alveoli (29). These results suggested that consolidation lesions are more invasive than only nodular/bronchiectasis lesions. Therefore, we classified the CT findings into three patterns (nodular/bronchiectasis lesions).

The number of MAC colonies in relevant lesions investigated by BMS was significantly larger than in the nondeteriorated group. This result suggests that the number of MAC colonies in relevant lesions is useful for predicting the short-term natural history of pulmonary MAC infection. The chest CT scan finding, too, was at least partly useful for predicting the short-term natural history of pulmonary MAC infection. The number of MAC colonies in patients with nodular/bronchiectasis lesions was significantly smaller than that in those with cavitary lesions. However, of 9 patients with consolidated lesions, 3 patients $(33 \%)$ were from the non-deteriorated group and $6(67 \%)$ were from the deteriorated group. Although consolidated lesions may be progressive, the number of MAC colonies is more useful for predicting deterioration. In fact, patients with consolidated lesions having more than $6.0 \times 10^{3} \mathrm{CFU} / \mathrm{mL}$ MAC colonies underwent deterioration in the present study. Okumura et al reported that fibrocavitary disease demonstrated a high risk for disease progression (26). All 8 patients with cavitary lesions in the present study deteriorated within 12 months. The number of MAC colonies in cavitary lesions was significantly greater than that in nodular/bronchiectasis lesions. Furthermore, the deteriorated patients with consolidated lesions showed high MAC colony numbers. Thus, the number of MAC colonies may predict deterioration more precisely than chest $\mathrm{CT}$ findings.

The number of MAC colonies collected by bronchial washing may also be useful for predicting the short-term natural history of pulmonary MAC infection, but in the present patients this was not significantly different among nodular/bronchiectasis lesions, consolidated lesions, and cavitary lesions. We suspect that the samples from peripheral lesions were not accurately collected because the washing fluid was minimal and sample quantity differed for each case. Therefore, the number of MAC colonies collected by bronchial washing does not reflect the difference between each lesion.

Because the natural history of pulmonary MAC infection is thought to vary widely, a diagnosis of pulmonary MAC infection does not necessarily imply a need for immediate treatment. The decision to provide treatment involves an observation period for both radiological and symptomatic progression (6). The present study suggests that cavitary and consolidated lesions that are evident on chest CT scans (especially with a high number of MAC colonies) indicate a 
high risk of progression for pulmonary MAC infection. Therefore, we suggest that patients with such chest CT scan findings begin therapy based on their clinical course to prevent further deterioration.

In conclusion, the number of MAC colonies in relevant lesions investigated by BMS was significantly larger in the deteriorated group than in the non-deteriorated group. Cavitary and consolidated lesions evident on chest CT scans displayed large numbers of MAC colonies and are thought to indicate high risk of deterioration. Although the sample size in the present study was small, BMS seems to be a useful tool to predict deterioration of pulmonary MAC infection.

\section{The authors state that they have no Conflict of Interest (COI).}

\section{Acknowledgement}

The authors wish to thank the staff from the Department of Laboratory Medicine of Shinshu University Hospital for MAC cultures.

\section{References}

1. Prince DS, Peterson DD, Steiner RM, et al. Infection with Mycobacterium avium complex in patients without predisposing conditions. N Engl J Med 321: 863-868, 1989.

2. Tanaka E, Amitani R, Niimi A, Suzuki K, Murayama T, Kuze F. Yield of computed tomography and bronchoscopy for the diagnosis of Mycobacterium avium complex pulmonary disease. Am J Respir Crit Care Med 155: 2041-2046, 1997.

3. Kubo K, Yamazaki Y, Hachiya T, et al. Mycobacterium aviumintracellulare pulmonary infection in patients without known predisposing lung disease. Lung 176: 381-391, 1998.

4. Yamazaki Y, Kubo K, Takamizawa A, Yamamoto H, Honda T, Sone S. Markers indicating deterioration of pulmonary Mycobacterium avium-intracellulare infection. Am J Respir Crit Care Med 160: 1851-1855, 1999.

5. Hunter AM, Campbell IA, Jenkins PA, Smith AP. Treatment of pulmonary infections caused by mycobacteria of the Mycobacterium avium-intracellulare complex. Thorax 36: 326-329, 1981.

6. Thomson RM, Yew WW. When and how to treat pulmonary nontuberculous mycobacterial diseases. Respirology 14: 12-26, 2009.

7. Wallace RJ Jr, Zhang Y, Brown BA, et al. Polyclonal Mycobacterium avium complex infections in patients with nodular bronchiectasis. Am J Crit Care Med 158: 1235-1244, 1998.

8. Ishizaka A, Watanabe M, Yamashita T, et al. New bronchoscopic microsample probe to measure the biochemical constituents in epithelial lining fluid of patients with acute respiratory distress syndrome. Crit Care Med 29: 896-898, 2001.

9. Ishizaka A, Matsuda T, Albertine KH, et al. Elevation of KL-6, a lung epithelial cell marker, in plasma and epithelial lining fluid in acute respiratory distress syndrome. Am J Physiol Lung Cell Mol Physiol 286: L1088-L1094, 2004.

10. Yanagi S, Ashitani J, Imai K, et al. Significance of human betadefensins in the epithelial lining fluid of patients with chronic lower respiratory tract infections. Clin Microbiol Infect 13: 63-69, 2007.

11. Nishimura $T$, Hasegawa $N$, Watanabe $M$, Takebayashi $T$, Tasaka $S$, Ishizaka A. Bronchoscopic microsampling to analyze the epithelial lining fluid of patients with pulmonary Mycobacterium avium complex disease. Respiration 76: 338-343, 2008.
12. Yamazaki K, Ogura $S$, Ishizaka A, Oh-hara $T$, Nishimura $M$. Bronchoscopic microsampling method for measuring drug concentration in epithelial lining fluid. Am J Respir Crit Care Med 168: 1304-1307, 2003.

13. Hasegawa $N$, Nishimura $T$, Watanabe $M$, et al. Concentrations of clarithromycin and active metabolite in the epithelial lining fluid of patients with Mycobacterium avium complex pulmonary disease. Pulm Pharmacol Ther 22: 190-193, 2009.

14. Watanabe M, Ishizaka A, Ikeda E, Ohashi A, Kobayashi K. Contributions of bronchoscopic microsampling in the supplemental diagnosis of small peripheral lung carcinoma. Ann Thorac Surg 76: 1668-1672, 2003.

15. Kahn N, Kuner R, Eberhardt R, et al. Gene expression analysis of endobronchial epithelial lining fluid in the evaluation of indeterminate pulmonary nodules. J Thorac Cardiovasc Surg 138: 474-479, 2009.

16. Sasabayashi M, Yamazaki Y, Tsushima K, Hatayama O, Okabe T. Usefulness of bronchoscopic microsampling to detect the pathogenic bacteria of respiratory infection. Chest 131: 474-479, 2007.

17. Herth FJ, Ernst A, Becker HD. Endobronchial ultrasound-guided transbronchial lung biopsy in solitary pulmonary nodules and peripheral lesions. Eur Respir J 20: 972-974, 2002.

18. Kurimoto N, Miyazawa T, Okimasa S, et al. Endobronchial ultrasonography using a guide sheath increases the ability to diagnose peripheral pulmonary lesions endoscopically. Chest 126: 959-965, 2004.

19. Griffith DE, Aksamit T, Brown-Elliot BA, et al; ATS Mycobacterial Disease Subcommittee; American Thoracic Society; Infectious Disease of Society of America. An official ATS/IDSA statement: diagnosis, treatment, and prevention of nontuberculous mycobacterial disease. Am J Respir Crit Care Med 175: 367-416, 2007.

20. Paone G, Nicastri E, Lucantoni G, et al. Endobronchial ultrasound-driven biopsy in the diagnosis of peripheral lung lesions. Chest 128: 3551-3557, 2005.

21. Lewin SR, Hoy J, Crowe SM, McDonald CF. The role of bronchoscopy in the diagnosis and treatment of pulmonary disease in HIV-infected patients. Aust N Z J Med 25: 133-139, 1995.

22. Wermert $\mathrm{D}$, Marquette $\mathrm{CH}$, Copin $\mathrm{MC}$, et al. Influence of pulmonary bacteriology and histology on the yield of diagnostic procedures in ventilator-acquired pneumonia. Am J Respir Crit Care Med 158: 139-147, 1998.

23. Wimberley N, Faling LJ, Bartlett JG. A fiberoptic bronchoscopy technique to obtain uncontaminated lower airway secretions for bacterial culture. Am Rev Respir Dis 119: 337-343, 1979.

24. Strumpf IJ, Feld MK, Cornelius MJ, Keogh BA, Crystal RG. Safety of fiberoptic bronchoalveolar lavage in evaluation of interstitial lung disease. Chest 80: 268-271, 1981.

25. Burns DM, Shure D, Francoz R, et al. The physiologic consequences of saline lobar lavage in healthy human adults. Am Rev Respir Dis 127: 695-701, 1983.

26. Okumura $\mathrm{M}$, Iwai $\mathrm{K}$, Ogata $\mathrm{H}$, et al. Clinical factors on cavitary and nodular bronchiectatic types in pulmonary Mycobacterium avium complex disease. Intern Med 47: 1465-1472, 2008.

27. Fujita J, Ohtsuki Y, Suemitsu I, et al. Pathological and radiological changes in resected lung specimens in Mycobacterium avium intracellulare complex disease. Eur Respir J 13: 535-540, 1999.

28. Moore EH. Atypical mycobacterial infection in the lung: CT appearance. Radiology 187: 777-782, 1993.

29. Jeong YJ, Lee KS, Koh WJ, Han J, Kim TS, Kwon OJ. Nontuberculous Mycobacterial pulmonary infection in immunocompetent patients: comparison of thin-section CT and histopathologic findings. Radiology 231: 880-886, 2004.

(C) 2011 The Japanese Society of Internal Medicine http://www.naika.or.jp/imindex.html 